\title{
Estudio del anticuerpo DOG1 en el diagnóstico de tumores del estroma gastrointestinal - GIST
}

\author{
The role of the DOG1 antibody in the diagnosis \\ of gastrointestinal stromal tumours - GIST
}

\author{
M.R. Mercado, M.L. Gómez Dorronsoro, A. Martínez-Peñuela Marco, \\ I. Rodríguez Pérez, I. Amat Villegas, J.M. Martínez-Peñuela Virseda
}

\section{RESUMEN}

Los tumores del estroma gastrointestinal (GIST) poseen mutaciones en los genes del receptor de la tirosín quinasa (RTKs) KIT y PDGFRA. La posibilidad de bloquear esta actividad ha significado una nueva esperanza terapéutica. El diagnóstico de GIST recae en la expresión inmunohistoquímica del c-KIT, pero un 4-15\% son c-KIT negativos (aún en presencia de mutación), y sin embargo estos pacientes podrían beneficiarse del tratamiento con inhibidores tirosín quinasa (TKIs).

El DOG1 es un nuevo anticuerpo cuya sensibilidad y especificidad parece ser superior o igual a la del c-KIT. El objetivo de este trabajo es evaluar la sensibilidad (Se) y especificidad (Sp) de DOG1 en GIST de tipo usual (c-KIT positivos), de tipo inusual (c-KIT negativos) y frente a otros tumores fusocelulares mesenquimales, y comparar la validez diagnóstica del DOG1 frente al cKIT.

Estudiamos 40 GIST, 39 c-KIT positivos y un c-KIT negativo. Se realizó un panel inmunohistoquímico con los anticuerpos: c-KIT, CD34, actina músculo liso, DOG1 y S100, en los GIST como en siete tumores fusocelulares.

La Se y Sp de GIST para DOG1 fue del 100 y $97,5 \%$ para c-KIT. La inmunoreactividad para DOG1 en todos los tumores fusocelulares fue negativa. La validez diagnóstica de DOG1 y C-KIT fue similar a la hora de detectar GIST y no GIST.

DOG1 es un marcador específico y sensible para el diagnóstico y diagnóstico diferencial de GISTs (es capaz de detectar algunos GIST sin mutación en RTK).

El DOG1 debería de formar parte del panel inmunohistoquímico para el diagnóstico de GIST.

Palabras clave. GIST. DOG1.

\begin{abstract}
Gatrointestinal stromal tumours (GIST) harbour oncogenic mutations in tyrosin kynases receptors (RTKs) including KIT and PDGFRA. The inhibition of this activity has been regarded as the primary target for the treatment of these patients. Diagnosis of GIST relies on c-KIT inmunoreactivity; however there is a $4-15 \%$ of GISTs that are C-KIT negative which may lead to underdiagnosis of GISTs and possible withholding of therapy.

The novel gene DOG1 has been found overexpressed in GISTs and has potential as a diagnostic marker for GISTs showing even more sensitivity (Se) and specificity (Sp) than c-KIT for the diagnosis of these tumors. In this study we compared the (Se) and (Sp) of DOG1 in typical and atypical GISTs (c-KIT positive or negative) with c-KIT and other mesenchymal neoplasms in the differential diagnosis of GISTs
\end{abstract}

We examined 40 GIST (39 showed inmunoreactivity for c-KIT and one was c-KIT negative) and another seven fusiform tumors. An inmunohistochemical panel was performed with c-KIT, CD34, smooth muscle actin, DOG1 and S100 antibodies on both types of neoplasms.

The overall Se and Sp of DOG1 and KIT in GISTs were nearly identical: 100 and 97,5\%. Negativity for DOG1 was observed in all fusiform mesenchymal neoplasms.

DOG1 is highly expressed in GIST and its expression seems quite specific for these tumours when the differential diagnosis includes another mesenchymal neoplasms.

DOG1 should be added to the diagnostic panel evaluating GISTs.

Key words. GIST. DOG1.
Servicio de Anatomía Patológica A. Complejo Hospitalario de Navarra

Recepción: 20 de enero de 2011

Aceptación provisional: 8 de febrero de 2011

Aceptación definitiva: 20 de mayo de 2011

\author{
Correspondencia \\ María del Rosario Mercado Gutiérrez \\ Servicio de Anatomía Patológica A. \\ Complejo Hospitalario de Navarra \\ Irunlarrea, 3 \\ 31008 Pamplona (Navarra) \\ E-mail: mr.mercado.gutierrez@navarra.es
}




\section{INTRODUCCIÓN}

Los tumores del estroma gastrointestinal (GIST) son las neoplasias mesenquimales más frecuentes del tracto gastrointestinal (TGI) y constituyen el 1\% de los cánceres primarios a este nivel. Se originan en las células intersticiales de Cajal (CIC) $)^{1,2}$ y su principal diagnóstico diferencial se plantea con otras neoplasias mesenquimales fusocelulares de similar localización (leiomiosarcoma, tumor desmoide, schwannoma, etc).

Tradicionalmente los GIST localmente avanzados se asociaban a un mal pronóstico. En casos de resección incompleta o enfermedad metastásica al momento de su presentación, la supervivencia promedio era inferior a un año, con una supervivencia a cinco años menor del 35\%. Además eran refractarios a tratamientos convencionales de quimioterapia, con una tasa de respuesta inferior al $10 \%$ sin mejoría en la supervivencia, y a la radioterapia, con tasas de respuesta menores al 5\%. De esta manera, hasta inicios del 2000 no existía una alternativa de tratamiento eficaz en etapas avanzadas ${ }^{3}$.

No fue hasta 1998 cuando Hirota y col$^{4}$ descubrieron el protooncogen KIT y Kindlom y col${ }^{5}$ las características inmunofenotípicas y morfológicas de la proteína c-KIT (CD 117) lo que originó un importante avance en la comprensión de estos tumores.

Las mutaciones del gen KIT serían el paso inicial para el desarrollo de GIST, que muestran mutación en un receptor de membrana con actividad tirosín quinasa, lo que condiciona su activación permanente y una proliferación celular no controlada. Mediante la mejor comprensión de la fisiopatología del GIST se han desarrollado una nueva clase de drogas antineoplásicas: los inhibidores de la tirosín quinasa (mesilato de imatinib).

Sabemos que el $90 \%$ de los GIST poseen mutaciones ya sea en el protooncogen KIT o en PDGFRA, y que la expresión de la proteína c-KIT ha demostrado ser un marcador relativamente específico para el diagnóstico de los GIST ${ }^{4,6-8}$.
Aunque el anticuerpo c-KIT es un marcador fiable para el diagnóstico de GIST, aproximadamente el 5\% de estos tumores presentan ausencia de expresión inmunohistoquímica para este marcador ${ }^{7,8}$. La ausencia de expresión inmunohistoquímica se asocia en su mayoría a la mutación del gen PDGFRA pero también a mutaciones del gen KIT en los exones 11 y $9^{8,9}$.

El $60-70 \%$ de los GIST c-KIT negativos son también PDGFRA negativos, lo que sugiriere la existencia de mecanismos adicionales aún desconocidos en su patogenia. Es por ello que surgió la necesidad de encontrar un marcador que pudiera identificar este tipo de tumores c-KIT negativos.

En el año 2004 West y col ${ }^{10}$ desarrollaron un nuevo antisuero que reacciona frente a un gen especifico del GIST y que codifica para un proteína hipotética (FLJ10261) que es altamente específica en el diagnóstico de GIST y cuya sensibilidad y especificidad parece ser que es igual o superior a la lograda con el c-KIT (CD 117) y CD 34: DOG111-13.

Son objetivos de este trabajo: evaluar la sensibilidad (Se) del anticuerpo DOG1 K9 en tumores del estroma gastrointestinal; evaluar la sensibilidad y especificidad (Sp) del anticuerpo DOG1 K9 frente a tumores fusocelulares mesenquimales: leiomiosarcomas, tumor fibroso solitario, schwanoma con los que se plantea el diagnóstico diferencial; y comparar la validez diagnóstica del anticuerpo DOG1 K9 frente a la del c-KIT.

\section{MATERIAL Y MÉTODOS}

Se seleccionaron un total de 42 tumores del estroma gastrointestinal (GIST) de los archivos del Servicio de Anatomía Patológica "A» del Complejo Hospitalario de Navarra (Pamplona), correspondientes a tumores diagnosticados entre enero de 1995 y enero de 2009, de los cuales se excluyeron dos casos por presentar escaso material. Se estudiaron GIST típicos (c-KIT o PGFRA positivos) (n=39) y sólo se encontró un GIST atípico (c-KIT negativo).

Se revisaron las preparaciones histológicas para clasificar los GIST en subtipos 
epitelioide, mixto o fusiforme según la morfología.

Se revisaron las historias clínicas correspondientes a los pacientes para recabar datos como: la localización del tumor, edad y sexo.

Se incluyeron áreas representativas de los tumores en microarrays de tejidos en cortes de seis micras donde se realizó un panel inmunohistoquímico con los siguientes anticuerpos: c-KIT (CD 117), CD 34, actina músculo liso, DOG1 K9 y S100, con los respectivos controles positivos y negativos. Los anticuerpos, clones, diluciones, condiciones de tratamiento y fuentes se resumen en la tabla 1.

Tabla 1. Panel de anticuerpos utilizados en el estudio

\begin{tabular}{|c|c|c|c|c|}
\hline Anticuerpo & $\begin{array}{c}\text { Recuperación } \\
\text { antigénica }\end{array}$ & Dilución & Clon & Fuente \\
\hline DOG1 & $\begin{array}{c}\text { Estándar } 60 \mathrm{~min} \\
\mathrm{CC} 1\end{array}$ & $1 / 100$ & $\mathrm{~K} 9$ & Menarini \\
\hline CD 117 (c-KIT) & $\begin{array}{c}\text { Estándar } 60 \mathrm{~min} \\
\mathrm{CC} 1\end{array}$ & $1 / 400$ & Policlonal & Dako \\
\hline CD 34 & $\mathrm{pH} 6-10 \mathrm{~min}$ & Prediluido & QBend/10 & Vitro \\
\hline S 100 & $\begin{array}{c}\text { Estándar } 60 \mathrm{~min} \\
\mathrm{CC} 1\end{array}$ & Prediluido & $4 \mathrm{C} 4.9$ & Menarini \\
\hline $\begin{array}{c}\text { Actina alfa } \\
\text { musculo liso (AML) }\end{array}$ & $\mathrm{pH} 6-10 \mathrm{~min}$ & $1 / 50$ & $\alpha \mathrm{M}-1$ & \\
\hline
\end{tabular}

Se valoró la tinción imunohistoquímica para DOG1 K9 y de los otros anticuerpos de manera semicuantitativa de la siguiente forma:

\section{Positividad $<25 \%$ de las células}

2. Positividad $25-50 \%$ de las células

3. Positividad $>50 \%$ de las células

Se realizó el mismo panel inmunohistoquímico en tumores fusocelulares de similar localización: tres leiomiosarcomas (en abdomen, duodeno y retroperitoneo), un schwannoma (en recto), una fibromatosis mesentérica (en duodeno). Se incluyeron además dos tumores fusocelulares de distinta localización: un tumor fibroso solitario (en pleura) y un sarcoma sinovial monofásico (en muslo). En estos tumores se realizó el panel inmunohistoquímico en cortes de $4 \mu \mathrm{m}$, desparafinados en xilol y rehidratados en alcohol.

Se realizó el análisis mutacional para el GIST c-KIT negativo. Se seleccionó una muestra representativa del tumor en parafina y se transfirió a un tubo eppendorf para la realización del estudio mutacional en los exones 18 y 12 de PDGFR-alfa y de los exones 11, 9, 13 y 17 de c-KIT en el Centro de Investigación Médica Aplicada de la Universidad de Navarra (Pamplona).

Todos los cálculos y el análisis estadístico se realizaron con SPSS V.15.0 (SPSS Inc. Illinois, EEUU).

Las variables cualitativas se describieron mediante frecuencias y porcentajes, mientras que las variables cuantitativas se describieron mediante media (desviación típica) si seguían una distribución normal y mediante mediana (rango intercuartílico: $\mathrm{P}_{25}-\mathrm{P}_{75}$ ) en caso contrario.

La validez diagnóstica se estableció a partir de los valores de sensibilidad y especificidad obtenidos de las tablas de contingencia. Los límites de los intervalos de confianza al 95\% (IC 95\%) se calcularon mediante el método exacto debido a las bajas frecuencias observadas.

La comparación del desempeño diagnóstico de dos de los anticuerpos estu- 
diados (DOG1 K9 y c-KIT) se llevó a cabo mediante el test de McNemar para datos pareados.

\section{RESULTADOS}

Se incluyeron 40 GIST de 40 pacientes, de los cuales 23 eran varones (57,5\%) y 17 mujeres $(42,5 \%)$, con una edad media de 63,2 (DS = 13,9) años (rango 24 a 85 años). El tamaño tumoral variaba desde 0,3 hasta
$11 \mathrm{~cm}$ de diámetro, con una mediana de 3,5 cm (1,85-5,75).

En estómago se localizaron 29 GIST (72,5\%), 10 en intestino delgado (25\%), incluyendo duodeno (2), yeyuno (7) e íleon (1), y un caso en sigma $(2,5 \%)$.

El aspecto microscópico varió de acuerdo al subtipo histológico. El $42,5 \%$ de los casos mostró una morfología fusiforme, el $40 \%$ se trató del subtipo epitelioide y $17,5 \%$ de tipo mixto (Fig.1).
$\mathbf{A}$

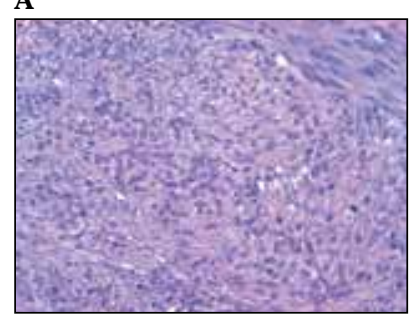

B



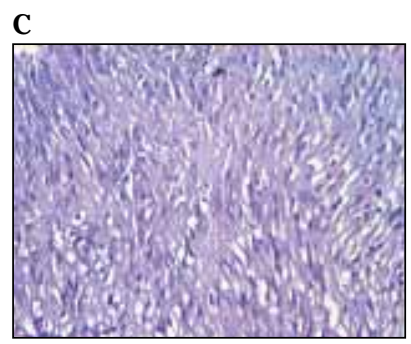

Figura 1. Morfología microscópica de los distintos tipos histológicos de GIST: A: epitelioide, B: fusocelular, C: mixto. HE x 40.

Los 40 GIST estudiados fueron inmunorreactivos para DOG1 K9, 39 para c-KIT $(97,5 \%), 35$ para CD $34(87,5 \%), 15$ para AML $(37,5 \%)$ y 8 para S100 (20\%).

En la mayoría de los GIST (75\%) la tinción para DOG1 fue citoplasmática e intensa en más del 50\% de las células (puntuación 3); en 9 casos (22,5\%) se observó una puntuación 2 ; en un caso (2,5\%) se observó inmunorreactividad en menos del $25 \%$ de las células (puntuación 1). El resto de resultados inmunohistoquímicos se resumen en la tabla 2 .

Tabla 2. Frecuencia de la intensidad para los anticuerpos DOG1, c-KIT, CD34, AML y S100 en GIST

\begin{tabular}{|l|c|c|c|c|c|}
\hline & DOG1 & c-KIT & CD 34 & AML & S100 \\
\hline Positivo & $40 / 40(100 \%)$ & $39 / 40(97,5 \%)$ & $35 / 40(87,5 \%)$ & $15 / 40(37,5 \%)$ & $6 / 40(15 \%)$ \\
\hline Puntuación 1 & $1 / 40(2,5 \%)$ & $3 / 39(7,6 \%)$ & $4 / 35(11,4 \%)$ & $8 / 15(53,3 \%)$ & $6 / 6(100 \%)$ \\
\hline Puntuación 2 & $9 / 40(22,5 \%)$ & $5 / 39(12,8 \%)$ & $5 / 35(14,2 \%)$ & $3 / 15(20 \%)$ & $0 / 6(0 \%)$ \\
\hline Puntuación 3 & $30 / 40(75 \%)$ & $31 / 39(79,4 \%)$ & $16 / 35(45,7 \%)$ & $4 / 15(26,6 \%)$ & $0 / 6(0 \%)$ \\
\hline Negativo & $0 / 40(0 \%)$ & $1 / 40(2,5 \%)$ & $5 / 40(12,5 \%)$ & $25 / 40(62,5 \%)$ & $34 / 40(85 \%)$ \\
\hline
\end{tabular}

En el único GIST c-KIT negativo se realizó el análisis mutacional de los exones 18 y 12 de PDGFR-alfa y de los exones 11, 9, 13 y 17 de c-KIT mediante PCR y secuenciación directa de los productos de amplificación, no habiéndose encontrado ningún tipo de mutación.

La tinción de DOG1 para los otros tumores fusocelulares incluidos (tres leiomiosarcomas, un schwannoma, una fibromato- 
sis mesentérica, un tumor fibroso solitario y un sarcoma sinovial monofásico) fue negativa.

En esta serie, todos los GIST presentaron inmunorreactividad frente al anticuerpo anti-DOG1 K9, mientras que los no GIST (7) no presentaron inmunotinción, presentando una sensibilidad y especificidad del $100 \%$, con unos intervalos de confianza al $95 \%$ de 90,07-100 y 59,0-100 respectivamente.

Los GIST inmunorreactivos frente a cKIT (39), fueron a su vez inmunoreactivos para DOG1 presentando una sensibilidad del $100 \%$ (intervalo de confianza al $95 \%$ de 90,75-100)

Todos excepto un GIST fueron inmunoreactivos para c-KIT ( $\mathrm{Se}=100 \%$ con un $\mathrm{IC}$ al 95\%: 86,84-99,94) y de los tumores no GIST ninguno fue inmunoreactivo para c-KIT (Sp $=100 \%$ con un IC 95\%: 59,0-100)

Al comparar la validez diagnóstica de anti-DOG1 y C-KIT se observó que ambos anticuerpos se comportan de forma casi idéntica a la hora de detectar GIST y no GIST ( $p=1,00$, McNemar) en esta serie.

\section{DISCUSIÓN}

Con el descubrimiento de las mutaciones en KIT y PDGFRA ${ }^{4,5}$ y el desarrollo de los TKI (inhibidores tirosina quinasa) para el tratamiento de los GIST, las opciones de tratamiento y pronóstico de los pacientes con enfermedad metastásica ha cambiado de manera radical, por lo que un diagnóstico adecuado de estos tumores -aún en ausencia de expresión de c-KIT- es esencial. El mesilato de imatinib (Glivec $®$ ) ha hecho posible el tratamiento de estos pacientes con enfermedad metastásica o localmente avanzada inoperable, alcanzando un control de la enfermedad en hasta un $85 \%{ }^{3,14}$.

Más del $90 \%$ de los GIST ocurren en adultos a partir de los 40 años de edad con una media de 63 años ${ }^{15}$ con un ligero predominio en varones. En nuestros casos la edad media de presentación fue también de 63 años, siendo más frecuentes en varones (57\%). La localización preferente es en estómago, intestino delgado, intestino grueso, esófago, epiplón, mesenterio y retroperitoneo ${ }^{3,15}$.

Nuestros casos presentaron una distribución similar de localizaciones (72,5\% en estómago, $25 \%$ en intestino delgado y $2,5 \%$ en intestino grueso sigmoide), no encontrando casos en otras localizaciones.

La mayoría de los GIST pueden ser diagnosticados a partir de una combinación de factores clínicos y anatomopatológicos, como son la localización, la apariencia histológica y la presencia de inmunorreactividad para c-KIT. Sin embargo, una proporción significativa de GIST (4-15\%) posee falta de expresión para c-KIT ${ }^{16,17}$. En estos casos sería necesaria la realización del estudio mutacional para KIT o PDGFRA para confirmar el diagnóstico de GIST; esto incrementa el coste y retrasa el diagnóstico de estos pacientes. DOG1 sería capaz de detectar estos GIST c-KIT negativos. Sin embargo, en los GIST c-KIT y DOG1 negativos se debe realizar un análisis mutacional para confirmar el diagnóstico.

DOG1 es una proteína de función desconocida que se expresa de manera selectiva en los GIST utilizando un perfil de expresión génica. Esta proteína se generó en suero de ratón; sin embargo, en estudios posteriores fue difícil generar en otros ratones a pesar de los intentos de inmunización de los mismos con el péptido DOG1 original ${ }^{13}$, por lo que se sintetizaron dos anticuerpos monoclonales (DOG1.1 y DOG1.3) que identifican diferentes secuencias del péptido DOG1. Ambos anticuerpos han mostrado mayor especificidad que c-KIT en la tinción de GIST, particularmente DOG1.1. Actualmente se dispone de un nuevo clon de este anticuerpo (K9) que ha logrado resultados similares a DOG1.1 y DOG1.3 en los trabajos realizados en este campo ${ }^{11,12}$.

Miettinem y col $^{13}$ describieron que el anticuerpo DOG1 identifica un $11 \%$ más de GIST que el c-KIT en tumores con mutación en KIT, sensibles a imatinib y c-KIT negativos, mientras que Liegl y col ${ }^{11}$ solo encontraron un $5 \%$ más. En nuestro estudio únicamente disponemos de un caso c-KIT negativo que fue intensamente positivo para DOG1, aunque en el estudio genético no presentó mu- 
tación ni para KIT (exones 11, 9, 13 y 17) ni para PDGFRA (exones 18 y 12).

Se ha descrito también que, a diferencia de los GIST mutados en KIT, los GIST que tienen mutación en PDGFRA no presentan inmunorreactividad para el c-KIT (o esta es débil) y la mayoría tienen una morfología epitelioide ${ }^{7,16}$, por lo que estos tumores tienden a ser diagnosticados como neoplasias de tipo epitelial. En estos casos la inmunoreactividad para DOG1 ha mostrado poseer una sensibilidad de alrededor de hasta un $90 \%{ }^{18}$. En nuestro caso c-KIT negativo se apreció una morfología epiteliode al igual que en estos estudios y no presentó inmunoreactividad para PDGFRA.

En el estudio de Espinosa y col$^{12}$, solo el $9 \%$ de los GIST que poseían mutación en el gen PDGFRA eran positivos para cKIT, mientras que la mayoría (78\%) fueron inmunorreactivos para DOG1; más aún, la tercera parte de los tumores con mutación en PDGFRA, c-KIT negativos e inmunorreactivos para DOG1.1 presentaron mutaciones que predecían una respuesta al imatinib.

Uno de los principales diagnósticos diferenciales de los GIST es el leiomiosarcoma. En nuestra serie los tres leiomiosarcomas estudiados fueron negativos para DOG1(K9). En otros estudios se ha descrito inmunorreactividad para DOG1 en estos tumores de manera aislada (alrededor de $0,3 \%)^{12}$

En los últimos años se ha descrito una alta sensibilidad y especificidad del DOG1 para GIST ${ }^{11-13,18}$. Espinosa y col $^{12}$, en los 1.367 tumores no GIST estudiados, solo encontraron inmunorreactividad para DOG1 en un leiomiosarcoma, un sarcoma sinovial y un melanoma desmoplásico. Además, se han observado escasos casos de carcinomas, melanomas y seminomas inmunorreactivos para DOG1 en comparación con el c-KIT. Ninguno de nuestros siete tumores no GIST mostró inmunorreactividad ni para DOG1 ni para c-KIT.

De los resultados de este trabajo se puede concluir que: DOG1 es un marcador altamente específico y sensible para el diagnóstico histológico de GIST y para realizar el diagnóstico diferencial con los tumores de morfología fusocelular.

La sensibilidad y especificidad de DOG1 y c-KIT no es significativamente distinta una de la otra y ambos se comportan de manera similar a la hora de diagnosticar un GIST.

El empleo de DOG1 en la práctica clínica complementa al c-KIT y debe formar parte del panel inmunohistoquímico para el diagnóstico de GIST.

\section{BIBLIOGRAFÍA}

1. Ramon y Cajal S. Sur les ganglions et plexus nerveux de l'intestine. Comp Rend Soc Biol Paris 1893; 45: 217-223.

2. Huizinga JD, Thuneberg L, Kluppel M, Malysz J, MiKkelsen HB, Bernstein A. W/kit gene required for interstitial cells of Cajal and for intestinal pacemaker activity. Nature. 1995; 373: 347349 .

3. Poveda A, Artigas V, Casado A, Cervera J, GarCía Del Muro X, Antonio López-Guerrero J et al. [Clinical practice guidelines in gastrointestinal stromal tumours (GEIS): update 2008]. Cir Esp 2008; 84 (Supl 1): 1-21.

4. Hirota S, Isozaki K, Moriyama Y, Hashimoto K, NISHIDA T, Ishiguro $S$ et al. Gain-of-function mutations of c-kit in human gastrointestinal stromal tumors. Science. 1998; 279: 577-580.

5. Kindblom LG, Remotti HE, Aldenborg F, MeisKINDBLOM JM. Gastrointestinal pacemaker cell tumor (GIPACT): gastrointestinal stromal tumors show phenotypic characteristics of the interstitial cells of Cajal. Am J Pathol 1998; 152: 1259-1269.

6. HeinRich MC, Corless CL, Demetri GD, Blanke CD, von Mehren M, Joensuu $H$ et al. Kinase mutations and imatinib response in patients with metastatic gastrointestinal stromal tumor. J Clin Oncol 2003; 21: 4342-4349.

7. Miettinen M, Wang ZF, Lasota J. DOG1 antibody in the differential diagnosis of gastrointestinal stromal tumors: a study of 1840 cases. Am J Surg Pathol 2009; 33: 1401-1408.

8. Debiec-Rychter M, Wasag B, Stul M, De Wever I, Van Oosterom A, HagemeiJer A et al. Gastrointestinal stromal tumours (GISTs) negative for KIT (CD117 antigen) immunoreactivity. J Pathol 2004; 202: 430-438.

9. Lasota J, Miettinen M. Clinical significance of oncogenic KIT and PDGFRA mutations 
in gastrointestinal stromal tumours. Histopathology 2008; 53: 245-266.

10. West RB, Corless CL, Chen X, Rubin BP, SubraMANIAN S, MONTGOMERY $\mathrm{K}$ et al. The novel marker, DOG1, is expressed ubiquitously in gastrointestinal stromal tumors irrespective of KIT or PDGFRA mutation status. Am J Pathol 2004; 165: 107-113.

11. Liegl B, Hornick JL, Corless CL, Fletcher CD. Monoclonal antibody DOG1.1 shows higher sensitivity than KIT in the diagnosis of gastrointestinal stromal tumors, including unusual subtypes. Am J Surg Pathol 2009; 33: 437-446.

12. Espinosa I, Lee CH, Kim MK, Rouse BT, SubramaNIAN S, Montgomery K et al. A novel monoclonal antibody against DOG1 is a sensitive and specific marker for gastrointestinal stromal tumors. Am J Surg Pathol 2008; 32: 210-218.

13. Miettinen M, Lasota J. Gastrointestinal stromal tumors-definition, clinical, histological, immunohistochemical, and molecular gene- tic features and differential diagnosis. Virchows Arch 2001; 438: 1-12.

14. Casali PG, Blay JY. Gastrointestinal stromal tumours: ESMO Clinical Practice Guidelines for diagnosis, treatment and follow-up. Ann Oncol 21 (Suppl 5): v98-102.

15. Parfitt JR, StreutKer CJ, Riddell RH, Driman DK. Gastrointestinal stromal tumors: a contemporary review. Pathol Res Pract 2006; 202: 837-847.

16. HuRlimann J, GARDIOL D. Gastrointestinal stromal tumours: an immunohistochemical study of 165 cases. Histopathology 1991; 19: 311-320.

17. RuBIN BP. Gastrointestinal stromal tumours: an update. Histopathology 2006; 48: 83-96.

18. LeE CH, Liang CW, Espinosa I. The utility of discovered on gastrointestinal stromal tumor 1 (DOG1) antibody in surgical pathology-the GIST of it. Adv Anat Pathol 2010; 17: 222-232. 